\title{
Transformation und neue Formen der Segregation in den Städten Lateinamerikas
}

\section{Karin Fischer, Christof Parnreiter, Wien}

\section{Einleitung}

Die Stadtentwicklung in Lateinamerika ist einem Wandel unterworfen, der durch die seit rund zwei Jahrzehnten ablaufende wirtschafts- und gesellschaftspolitische Transformation verursacht wird. Diese Annahme bildet die Kernthese eines Forschungsprojektes am Institut für Stadt- und Regionalforschung der Österreichischen Akademie der Wissenschaften. Analysiert wird, wie sich die Abkehr von der importsubstituierenden Industrialisierung und die vertiefte Integration Lateinamerikas in den Weltmarkt (Forcierung der Exportproduktion, Öffnung des Binnenmarktes für Importe, Werben um ausländische Investitionen) sowie die Neugestaltung der Beziehungen zwischen Staat, Kapital und Arbeit (Privatisierungen, Flexibilisierung des Arbeits- und Bodenmarktes) auf die Stadtentwicklung auswirken. Insbesondere geht es dabei um zwei Aspekte, die den Urbanisierungsprozess über Jahrzehnte maßgeblich geprägt haben, nämlich die Funktion der Hauptstadt im nationalen Städtesystem und die Dynamik der sozialräumlichen Entwicklung im Inneren der Städte (vgl. PARnReITER 2001, 2002).

Der zweite Punkt bildet das Thema dieses Aufsatzes. Bilden sich in den Städten Lateinamerikas tatsächlich, wie von manchen Forschern argumentiert wird, neue Formen sozialräumlicher Segregation heraus, und wenn ja, wodurch sind sie gekennzeichnet? Zweitens: Besteht ein Zusammenhang zwischen dem Entstehen neuer Segregationsformen und den Transformationsprozessen?

Zunächst wird skizziert, zu welchen Ergebnissen die lateinamerikanische Segregationsforschung bis zum Einsetzen der Transformationsprozesse gelangt ist. Dann werden Arbeiten vorgestellt, die einen Wandel in den Segregationsformen identifizieren und mögliche Gründe für diese Veränderungen genannt. Abschließend werden Forschungsaufgaben, insbesondere im Zusammenhang mit den condominios (gated communities) thematisiert.

\section{Segregation und Stadtmodelle bis in die 1990er Jahre}

Im vorliegenden Beitrag wird unter räumlicher Segregation die disproportionale Verteilung der Wohnorte sozialer Gruppen verstanden. Diese «residenzielle Segregation» (vgl. dazu Dangschat 1997) wird erstens durch die räumliche Konzentration sozialer Schichten in städtischen Teilgebieten bestimmt und zweitens durch den Grad an sozialer Homogenität innerhalb derselben (Sabatini et al. 2001). Diese beiden Definitionskriterien bezeichnen jeweils eine bestimmte Seite der sozialräumlichen Entmischung: So können reiche Bevölkerungsschichten zwar räumlich konzentriert sein (das erste Merkmal trifft stark zu), ihr Gebiet aber zugleich mit anderen sozio-ökonomischen Gruppen teilen (das zweite Kriterium trifft nur wenig zu). Dieser doppelte Begriffsinhalt ist von analytischer Bedeutung, wie hier im Zusammenhang mit den sozialräumlichen Veränderungen in Lateinamerikas Städten gezeigt werden wird.

Zur Entstehung der disproportionalen Verteilung gibt es in der Segregationsforschung bis dato kein überzeugendes Konzept (Dangschat 1997: 643; SchteINGART 2001: 19). Es ist allerdings zu vermuten, dass es sich um einen wechselseitigen Prozess handelt, dass also - idealtypisch gesehen - bestimmte Bevölkerungsgruppen spezifischen Wohnorten zugewiesen werden, was wiederum dazu führt, dass diese Wohnorte nur noch von der jeweils konkreten sozialen Gruppe in Betracht gezogen werden.

Die deutschsprachige Segregationsforschung führte zur Erstellung von Strukturmodellen für die lateinamerikanische Stadt (für einen Überblick über die Modelle von Borddorf, Mertins, Gormsen sowie Bähr \& MerTins siehe Wilhelmy \& BorSDORF 1984: 181-190; für eine Weiterentwicklung siehe BORSDORF et al. in diesem Band). Trotz zum Teil erheblicher Unterschiede ist diesen Arbeiten gemeinsam, dass sie ursprünglich eine eindeutige Zuordnung der sozialen Gruppen zu bestimmten städtischen Zonen (seien es Ringe, Sektoren, Kerne oder Zellen) zeigten. Eine solche Klarheit liegt natürlich im Wesen eines jeden Modells, andererseits wurde sie durch den impliziten oder expliziten Bezug zu den recht schematischen Arbeiten der Chicagoer Schule der Sozialökologie begünstigt. In mehreren Überarbeitungsschritten gewannen die Modelle zur lateinamerikanischen Stadtstruktur an Komplexität, die Grundidee einer relativ deutlichen Differenzierung der Wohngebiete unterschiedlicher sozialer Gruppen aber wurde beibehalten.

Diese Idee bildete auch im angelsächsischen Sprachraum das Leitmotiv der auf Lateinamerika bezogenen 
Stadtforschung (zusammenfassend siehe PORTES 1989: 16). GilBeRT (1994: 84) brachte dieses Einvernehmen auf den Punkt:

"As a general rule, rich and poor live in different areas of the Latin American city (...). The rich choose their preferred locales and the poor occupy the land that is left over, usually in the least attractive parts of the city".

\section{Neue Formen sozialräumlicher Segregation und ihre (möglichen) Gründe}

Neue Forschungsarbeiten stellen das kohärente Bild der lateinamerikanischen Stadt in Frage (für Überblicke siehe Portes et al. 1997; LUNGo \& Baires 2001; für Fallstudien siehe CALDEIRA 1999; ClichEvSKY 2000; Rubalcava \& Schteingart 2000; Sabatini 2000; Ciccolella 1999 Sabatini et al. 2001; SchteingarT 2001; Torres 2001; Ciccolella \& Mignaqui 2002). Zwar leidet die jüngere Segregationsforschung in Lateinamerika generell an einem Mangel an empirischen Untersuchungen und auch an einer konzeptionell-theoretischen Schwäche (SABATINI et al. 2001; SCHTEINGART 2001), dennoch konnte sie mit ihren Analysen auf eine Veränderung der Segregationsformen aufmerksam machen. Den impliziten oder expliziten Referenzpunkt dieser Studien bilden die Transformations- und Globalisierungsprozesse der letzten zwei Jahrzehnte.

Im Hinblick darauf, dass Änderungen in der sozialräumlichen Struktur nur äußerst langsam vor sich gehen, werden die neuen Entwicklungen als Tendenzen gefasst, die historische Segregationsmuster nicht außer Kraft setzen. Trotz dieser Einschränkung und erheblicher regionaler Besonderheiten werden zwei signifikante Veränderungen festgestellt: Einerseits wird beobachtet, dass sich in städtischen Teilgebieten eine größere soziale Komplexität herausbildet, weil «arm» und «reich» in kleineren Räumen aufeinander treffen. Dies wird als ein Trend zur Verschärfung von Segregation interpretiert, weil die jeweiligen sozialen Gruppen ihre Anstrengungen zur Abgrenzung verstärken, um die soziale Distanz zu wahren. Andererseits führt diese Entwicklung zu einer größeren sozialen Inhomogenität in den betreffenden städtischen Teilräumen, was, gemäß der oben angeführten Definition, als Abnahme der Segregation gedeutet werden kann.

Diese Veränderungen werden in den zitierten Studien in BORSDORF et al. (in diesem Band) auf unterschiedliche Wanderungsprozesse in den Städten zurückgeführt. Erstens tragen intraurbane Migrationen zu kleinräumigeren Segregationsmustern bei. Diese Wanderungsprozesse können unterschiedliche Richtungen einschlagen: Die verarmte Mittelschicht siedelt sich in Gebieten an, die traditionell von der städtischen Unterklasse bewohnt werden; umgekehrt suchen alte und neue Arme die Nähe zu potenziellen Beschäftigungsmöglichkeiten und damit auch zu Mittelschichtund Oberschichtgebieten.

Neben Verarmungsprozessen trägt auch die Errichtung großflächiger Wohnprojekte zu einer Änderung residenzieller Segregationsmuster bei. Der Zuzug von Ober- und Mittelschichtfamilien in ärmere innerstädtische Gebiete, aber auch die Ansiedlung mittlerer Einkommensschichten in der Nähe traditioneller Oberschichtviertel erfolgt oft in der Form von condominios. Diese schotten sich sowohl durch bauliche Maßnahmen (Mauern, Zäune) als auch durch private Sicherheitsdienste von ihrer Umgebung ab (siehe die Beiträge von BORSDORF et al.; COY \& PöhleR; JanoschKa; Kanitscheider; Kohler in diesem Band). Die größere physische Nähe geht deshalb nicht einher mit einer stärkeren Durchmischung der Wohnbevölkerung. Kleinräumigere residenzielle Segregation ist also nicht gleichbedeutend mit sozialer Integration, vielmehr wird Ungleichheit auf kleinerem Raum produziert und reproduziert. Reichtumsinseln entstehen inmitten alter und neuer Armut. Allerdings prägen sozial homogene Armutszonen weiterhin das Bild vieler lateinamerikanischer Großstädte: Die traditionellen Elendsquartiere sind als Folge der generellen Verarmung stark angewachsen.

Zweitens wird, neben der intraurbanen Migration, eine Auswanderung wohlhabender Schichten in den suburbanen Raum festgestellt. Dort, an der «ehemaligen» Peripherie, werden in vielen lateinamerikanischen Städten durch den Bau von großflächigen condominios attraktive Wohnmöglichkeiten geschaffen. Damit treffen Familien der Mittel- und Oberschicht entweder mit ländlicher, in der Regel armer Bevölkerung zusammen, oder sie okkupieren Raum, der lange Zeit in erster Linie billiger und zum Teil subventionierter Wohnraum für Arbeiter und Migranten darstellte. Beides verstärkt den Trend zu einem dichteren Nebeneinander von «arm» und «reich».

Die Triebkräfte für diese Entwicklungen liegen in der ökonomischen Krise und der Transformation. $\mathrm{Zu}$ den bereits angesprochenen Verarmungsprozessen kommen die - wegen der Privatisierung - gestiegenen Transportkosten sowie eine Verteuerung der Bodenpreise an den Stadträndern. Weil diese zunehmend in den Immobilenmarkt einbezogen werden (u.a. wegen des starken Flächenwachstums der Städte), können sich die ärmsten Bevölkerungsschichten selbst an der Peripherie Wohnen nicht mehr leisten. Sie ziehen zurück in zentral gelegene Unterschichtgebiete oder sind dazu gezwungen, in noch weiter von der Kernstadt 
entfernten Zonen nach Wohnmöglichkeiten zu suchen. Die Expansion des Stadtareals wird auch durch den steigenden Bedarf an Büroflächen bewirkt, der sich aus der mit der Transformation einhergehenden Zunahme von Finanz- und Produktionsdienstleistungen ergibt. Neue Bürokomplexe werden oftmals an der «alten» Peripherie angesiedelt, wo urbane Subzentren entstehen, die ihrerseits neue Wohnbevölkerung anziehen.

Ein weiterer räumlicher Aspekt der sozioökonomischen Transformation ist, dass sich die Standorte der während der importsubstituierenden Industrialisierung aufgebauten, im Zeitalter der Globalisierung aber entwerteten Industrien in einem Prozess der Zerstörung und Aufwertung befinden. Vor allem in den Innenstadtgebieten werden die brachliegenden Fabriken und Häuser in billige Wohnquartiere umfunktioniert; in anderen Teilen werden Appartementhäuser für Mittelklassen, meist in Form von condominios, errichtet.

Ausdruck und zugleich Triebkraft der sozioökonomischen Transformation ist der gestiegene Handlungsspielraum des privaten Immobiliensektors. In vielen Fällen bewirkte das Zusammenspiel von profitablen Anlagebedingungen für in- und ausländische Kapitaleigner mit der Liberalisierung der Bodenpolitik einen Immobilienboom, der sich in Investitionen in großflächige Wohnprojekte, Hotels, Entertainmentund Einkaufszentren sowie in den Neubau resp. Ausbau des privat konzessionierten städtischen Autobahnnetzes niederschlug. Darüber hinaus konnten mit dem Abbau klientelistischer Politik und der geschmälerten Rolle der Kommunen im Wohnbau auch die peripheren Armutszonen in den formellen Grundstücksmarkt einbezogen werden. Durchsetzungsfähige «developers» kaufen zu den in Armengegenden «ortsüblichen» Preisen Land, um darauf großflächige condominios für die Ober- und Mittelschicht zu errichten. Um die soziale Distanz zur ansässigen Bewohnerschaft zu wahren, werden die Zugezogenen durch Mauern und Zäune abgeschirmt. Der private Immobiliensektor zählt somit zu den wichtigsten Akteuren bei der (Um-)Gestaltung des städtischen Raums und der Etablierung neuer, kleinräumigerer Segregationsformen.

Zusammenfassend halten wir fest, dass die jüngere Segregationsforschung vermuten lässt, dass die lange angenommene klare räumliche Differenzierung der Wohngebiete unterschiedlicher sozialer Gruppen dem komplexen Bild der gegenwärtigen Stadtentwicklung in Lateinamerika nicht (mehr) entspricht. Die neuen Tendenzen deuten vielmehr auf das Entstehen einer multi-fragmentierten Stadt hin.

\section{Condominios und «multi-fragmentierte» Stadt: eine Forschungsagenda}

Condominios spielen eine wichtige Rolle in der Entstehung dieser «multi-fragmentierten» Stadt. Sie ermöglichen die Aufhebung der räumlichen Distanz zwischen den gesellschaftlichen Gruppen durch «Bunkerisierung» - Mauern, Überwachungskameras und Sicherheitsdienste übersetzen die sozialen Unterschiede in räumliche Barrieren. Condominios stellen mit ihrer Abschottung nach außen und ihrer Homogenität nach innen eine bauliche und soziale Form dar, die den Wandel der Segregationsmuster von einer relativ großflächigen Aufteilung der Stadt in Armen- und Reichenviertel hin zu kleinräumigeren, aber gleichzeitig intensivierten Formen der Segregation ermöglichen.

Darüber hinaus tragen sie zur Privatisierung öffentlichen Raumes bei. Auch wenn die rechtlichen Grundlagen von condominios von Land zu Land variieren, so ist doch in ganz Lateinamerika zu beobachten, dass gegen das oder am Gesetz vorbei öffentliche Straßen abgesperrt oder Gemeinflächen in privaten Besitz genommen werden. Diese Aneignung öffentlichen Raums führt, gemeinsam mit der «Bunker-Architektur» und dem massenmedial getragenen Diskurs der Angst, zur Frage, ob die massive Ausbreitung der condominios nicht das Ende der traditionellen lateinamerikanischen Stadt markiert, die durch dichte soziale Beziehungen und reges öffentliches Leben charakterisiert ist.

Die Untersuchung der condominios steht allerdings erst an ihrem Beginn. Die Forschungsagenda umfasst erstens Fragen der rechtlichen Bestimmung, Definition und Typisierung. Zweitens erlaubt der derzeitige Forschungsstand keine quantitative Einschätzung und damit auch keine qualitative Beurteilung des Phänomens. Fast vollständig ausgeklammert blieb in der bisherigen Forschung auch, welche Rolle condominios außerhalb der Metropolen, also in sekundären Millionenstädten oder gar in Klein- und Mittelstädten spielen.

Drittens: Warum kommt es zum condominio-Boom und wer sind die zentralen Akteure? Hinsichtlich möglicher struktureller Faktoren sind an prominenter Stelle die Auswirkungen der Deregulierung des Immobilienmarktes zu analysieren, die Immobilienfirmen zu treibenden Akteuren der Stadtentwicklung werden ließen. Voraussetzung dafür sind - neben der Deregulierung - der Rückzug der Kommunen und des Staates aus der Wohnungspolitik sowie das Vorhandensein von «überschüssigem» Kapital (vgl. Arrighi 1994), das in Luxuswohnbau angelegt wird. 
Einer genauen Analyse bedarf auch die Politik gegenüber den condominios. Einerseits greifen die Kommunen aktiv in den Bauboom ein, indem sie «developers» zu Investitionen bewegen in der Hoffnung, sich Konkurrenzvorteile im globalen, nationalen und innerstädtischen Standortwettbewerb zu verschaffen (vgl. Harvey 1989). Andererseits gilt es das Zusammenspiel von mächtigen privaten Akteuren (z.B. Immobilienfirmen) und der Politik zu untersuchen. $\mathrm{Ob}$ institutionelle Regulierungen von Stadt und Staat die condomino-Bautätigkeit, die Privatisierung des öffentlichen Raumes oder eigene Regeln innerhalb der condominios erlauben, dulden oder verbieten, hat konkrete Auswirkung auf die Stadtentwicklung.

Ein anderer wichtiger Aspekt ist die von den USA ausgehende Globalisierung bestimmter Bilder vom «schönen Wohnen», die private Immobilienfirmen vorantreiben und sich zu Nutze machen. Zu diesen Vorstellungen gehört einerseits das Vorhandensein einer bestimmten Luxusinfrastruktur (z.B. Swimmingpools oder Golfplätze), andererseits wird das Bedürfnis, der Hektik, dem Lärm und Autoverkehr der Großstadt zu entkommen, verknüpft mit Reminiszenzen an das «ruhige Dorfleben», das (angeblich) in condominios zu finden ist.

Schließlich sind auch Fragen der Sicherheit zu den strukturellen Triebkräften des condominio-Booms zu zählen. Gerade in diesem Bereich sind aber sorgfältige Studien erforderlich, um die Bedrohung durch Kriminalität von einer massenmedial bewusst erzeugten Atmosphäre der Angst zu unterscheiden, die unter anderem bezweckt, eine Nachfrage nach «sicherem» Wohnraum zu erzeugen. Dadurch wird die Abschottung der Ober- und Mittelschicht nicht nur legitimiert, sondern auch verstärkt: Wer im «Sicherheitsghetto» lebt und die realen Kontakte mit der Außenwelt einschränkt, wird empfänglicher für Angstphantasien - «die Anderen» werden zunehmend als Bedrohung wahrgenommen.

Hinsichtlich der Nachfrage ist weiter genauer zu untersuchen, warum Menschen in condominios ziehen. $\mathrm{Zu}$ den bereits genannten Gründen (Lifestyle, Ruhe, Sicherheit) kommt das Bedürfnis, unter «seinesgleichen» zu leben - Sicherheit also nicht nur vor realen und eingebildeten Gefahren zu finden, sondern auch vor der Herausforderung, in der Stadt mit Fremdartigem und Chaos konfrontiert zu sein.

Ein vierter Punkt auf der Forschungsagenda betrifft die internen Prozesse in den condominios. Soziale Beziehungen zwischen den Bewohnern sind ebenso zu analysieren wie ihre Außenkontakte - woher kommen beispielsweise Freunde, Putzfrauen und Gärtner? Untersuchenswert ist auch, ob sich ein
Zusammenhang zwischen der Zugehörigkeit zu einer bestimmten sozialen Schicht und einem konkreten Typ von condominio feststellen lässt. Schließlich ist nach den Aktionsräumen zu fragen: Leben die Bewohner wirklich in Ghettos (Wohnung, Büroturm, Supermarkt), die miteinander nur durch die Reise im Privatwagen verbunden sind?

Fünftens muss die condominio-Forschung in die Segregations- und Stadtforschung eingebettet werden. Der hier postulierte Zusammenhang zwischen der Ausbreitung von condominios und dem Entstehen neuer Formen kleinräumiger Segregation, die ihrerseits zu einer «multi-fragmentierten» Stadt führt, bedarf sowohl weiterer empirischer Belege als auch einer tieferen theoretischen Konzeptualisierung. Zu untersuchen ist etwa, ob und welche Interaktionen es zwischen den condominios und anderen Stadtteilen gibt. Bieten condominios den Menschen in den sie umgebenden Armenvierteln bezahlte Erwerbsmöglichkeiten? Wie entwickelt sich der «unverbunkerte Rest» der Stadt, der immer noch die meisten Bewohner beherbergt und die größte Fläche einnimmt? Welche Auswirkungen haben condominios auf die urbane Infrastruktur oder den Verkehr? Was passiert mit einer Stadt, wenn sie zunehmend in Fragmente zerfällt und öffentlicher Raum privat angeeignet wird? Welche Rückwirkungen hat die Privatisierung der Wohnungspolitik auf die Stadtentwicklung?

\section{Weitere Herausforderungen für die lateinamerika- nische Segregationsforschung}

Um die condominio-Forschung in die Stadtforschung einbetten und die Diskussion um neue Formen der Segregation vertiefen zu können, müssen konzeptionelle und empirische Herausforderungen bewältigt werden.

Erstens: Trotz aufschlussreicher Studien bleibt bislang offen, ob und wie die hier beschriebenen Entwicklungen mit den wirtschafts- und gesellschaftspolitischen Transformationen der letzten zwei Jahrzehnte zusammenhängen. Zahlreiche Indizien legen eine solche Verknüpfung nahe: Das Erstarken des Immobiliensektors beispielsweise ist nicht ohne eine vorhergehende Deregulierung des Bodenmarktes oder ohne die Zuflüsse «globalen» Kapitals denkbar. Oder: Die Verarmung breiter Schichten, die oft als ein wichtiger Grund für die Herausbildung neuer Segregationsmuster genannt wird, ist ebenso eine Folge der neoliberalen Strukturanpassung wie das Entstehen einer neuen, kaufkräftigen Schicht, die sich die condominios leisten kann. Wie allerdings das Erstarken des Immobiliensektors oder eine Vertiefung der sozialen Polarisierung mit der Herausbildung 
neuer räumlicher Spaltungen zusammenhängt, ist bislang weder theoretisch noch empirisch aufgearbeitet worden.

Zahlreiche Forscher weisen denn auch auf Probleme der Segregationsforschung hin, die in der schwachen theoretischen Fundierung vieler Arbeiten liegen. Insbesondere wird ein mangelhaftes Verständnis des Zusammenhangs zwischen sozialer und räumlicher Ungleichheit kritisiert. Beispielsweise wird oft a priori angenommen, dass die - gut dokumentierte - Zunahme sozialer Ungleichheit in Folge der Transformationsprozesse unvermeidlich zu einer ansteigenden «sozialräumlichen Segregation» führe. Obwohl dies nicht auszuschließen ist, fehlen dafür die Belege. Zudem sind die aktuellen Entwicklungstendenzen in lateinamerikanischen Städten zu komplex, um eine symmetrische Beziehung zwischen sozialer und räumlicher Polarisierung anzunehmen. Residenzielle Segregationsmuster entstehen nicht als automatischer Reflex auf soziale Ungleichheit (Dangschat 1997; Sabatini et al. 2001; SCHTEINGART 2001).

Zweitens: Kann dem erwähnten Mangel an quantitativen und qualitativen Untersuchungen durch künftige Studien auch abgeholfen werden, so müssen dabei auch methodische Probleme gelöst werden. Zum einen reicht die quantitative Messung von Ungleichheit (auf der Basis von Zensusdaten) nicht aus, um die sozialen Auswirkungen neuer residenzieller Segregationstendenzen adäquat in den Blick zu bekommen. Wie Menschen subjektiv die beiden «objektiven Dimensionen» von Segregation (die Konzentration von sozialen Gruppen und die soziale Homogenität in Wohngebieten) wahrnehmen, prägt das Gefühl von sozialem Ein- oder Ausschluss. Erst qualitative Interviews ermöglichen Schlussfolgerungen über die konkret erfahrbaren Auswirkungen räumlicher Restrukturierung.

Zum anderen ist auch die quantitative Segregationsforschung mit methodischen Problemen konfrontiert, da ihre zentralen Elemente (wie Armuts- und Reichtumsindikatoren oder räumliche Untersuchungseinheiten) in der Regel nicht von den Forschern, sondern von den statistischen Instituten bestimmt werden. Boltvinik (1999) zeigt am Beispiel Mexikos, dass Zensusdaten zur Wohnungsausstattung, zum Einkommen oder zur Bildung nur ein eingeschränktes Verständnis von Armut oder Reichtum ermöglichen. Zudem weisen selbst diese Indikatoren Mängel auf, weil Einkommen nur bis zu einer Obergrenze von fünf Mindestlöhnen erhoben werden und diese fünf Mindestlöhne nur knapp oberhalb der Armutsgrenze liegen. Folglich erlauben es die Zensusdaten nicht, die Mittelschicht von der Oberschicht anhand der Einkommen zu unterscheiden. Zudem zieht das mexikanische Statistikinstitut den erheblichen Reallohnver- fall der letzten 20 Jahre nicht in Betracht, weshalb ein historischer Vergleich der Einkommen zu einer Verzerrung führt.

Ein weiteres methodisches Problem betrifft die Definition der Untersuchungseinheit. Wenn die Homogenität in Wohngebieten ein zentrales Kriterium zur Definition von residenzieller Segregation ist, dann ist die Größe der Untersuchungseinheit von entscheidender Bedeutung für das Forschungsergebnis. Im Regelfall können Segregationsforscher ihre Untersuchungseinheit aber nicht wählen. Sie wird ihnen, ebenso wie die Bestimmung von Armut und Reichtum, von den nationalen Statistikinstituten vorgegeben, weil diese bestimmen, auf welchem Maßstab Daten erhoben werden. Der gewählte Maßstab allerdings hat gravierende Auswirkungen auf das Bild, das wir uns von der Segregation machen können: je größer die gewählte Untersuchungseinheit, desto wahrscheinlicher ist es, dass der Bereich als homogen erscheint, weil feine Unterschiede innerhalb des Gebietes statistisch nivelliert werden (Sabatini et al. 2001).

Die jüngste Arbeit von Rubalcava und Schteingart (2000), die auf den mexikanischen Zensusdaten von 1990 basiert, zeigt diese Problematik exemplarisch auf. Die Einführung einer neuen, weniger als 6.000 Personen umfassenden statistischen Zähleinheit erlaubt es, Daten kleinräumig zu erfassen. Damit können erstmals kleinräumige Unterschiede in der demographischen, sozialen und wirtschaftlichen Struktur von Mexico City festgestellt werden. Dies erlaubt es zu zeigen, dass eine beträchtliche Zahl von Haushalten der Mittelschicht und sogar der Unterschicht in wohlhabenden Gebieten anzutreffen sind, und zwar sowohl im Stadtzentrum als auch in den traditionellen Oberschichtvierteln im Westen der Stadt. Allerdings kann nicht beurteilt werden, ob es sich bei der so identifizierten kleinräumigen residenziellen Segregation um die Folge einer neuen Dynamik handelt, oder ob das Ergebnis «nur» auf einer Verbesserung der Erhebungsmethoden beruht - Vergleichsdaten aus der Zeit vor 1990 sind nicht verfügbar. Jedenfalls stellt die Studie von Rubalcava und Schteingart die bislang dominierende Sichtweise einer klaren räumlichen Trennung der sozialen Gruppen in Frage.

\section{Fazit}

Die traditionell auf Armutszonen und die Unterschicht fokussierte Stadtforschung hat durch die Beschäftigung mit den Wohnformen der reichen und sozial aufsteigenden Schichten auf neue und differenzierte Formen residenzieller Segregation aufmerksam gemacht. Diese Arbeiten lassen vermuten, dass das charakteristische, großflächige Segregationsmuster in lateinamerika- 
nischen Städten sich zugunsten einer kleinräumigeren, aber intensiveren Segregation verändert.

Die Neuheit dieser Entwicklung besteht in zwei unterschiedlichen Tendenzen: Die kleinmaßstäbliche soziale Entmischung geht einerseits zurück, weil bestimmte Stadträume zu Wohngebieten unterschiedlicher sozialer Gruppen werden, wodurch sich die physische Distanz zwischen diesen verringert. Andererseits wird die residenzielle Segregation vertieft, weil die Wohngebiete im Inneren sozial homogen, nach außen aber scharf abgegrenzt sind. Die soziale Distanz bleibt somit trotz räumlicher Nähe erhalten oder wird sogar vergrößert.

Damit zeigen lateinamerikanische Städte heute ein komplexeres Bild als vor zwei Jahrzehnten, wozu neben dem condominio-Boom auch das Entstehen von großflächigen Büro-, Entertainment- und Einkaufszentren außerhalb der traditionellen Oberschichtgebiete (oft als Subzentren an den ehemaligen Stadträndern) beitragen. Beides, die Prozesse der Dezentralisierung und der kleinräumigeren, gleichzeitig aber intensiveren Segregation, deuten auf die Entstehung multi-fragmentierter Stadtstrukturmuster hin.

Neben sorgfältigen Analysen darüber, inwieweit eine solche multi-fragmentierte Stadt, die aus zahlreichen funktions- und sozialräumlichen Teilen zusammengesetzt ist, durch ökonomische Transformationsprozesse geformt wird, erfordert die Beschäftigung mit den widersprüchlichen und komplexen Segregationsformen den Blick aufs Ganze: Die Frage, ob die identifizierten Entwicklungen Chancen für eine stärkere soziale Integration ihrer Bewohner bieten oder es im Gegenteil zu einer Vertiefung des Ghetto-Effektes kommt, weil «Parallelgesellschaften» auf kleinerem Raum unverbunden nebeneinander existieren, verweist direkt auf das Erscheinungsbild der lateinamerikanischen Stadt in der Zukunft.

\section{Dank}

Diese Arbeit wurde finanziert durch den Fonds zur Förderung wissenschaftlicher Forschung (Nummer P 14883).

\section{Literatur}

Arrighi, G. (1994): The Long Twentieth Century. Money, Power, and the Origins of Our Times. - London: Verso.

Boltvinik, J. (1999): Pobreza y distribución del ingreso en México. - México D.F.: Siglo XXI.

Caldeira, T. (1999): Fortified Enclaves: The New Urban Segregation. - In: Holston, J. (Hrsg.): Cities and
Citizenship. - Durham, NC: Duke University Press: 114-138.

CicCOlella, P. (1999): Globalización y dualización en la Región Metropolitana de Buenos Aires: Grandes inversiones y reestructuración socioterritorial en los años noventa. - In: EURE, Revista Latinoamericana de Estudios Urbano Regionales 25/76, Santiago de Chile: 5-27.

Ciccolella, P. \& I. Mignaqui (2002): Buenos Aires: Sociospatial Impacts of the Development of Global City Functions. - In: Sassen, S. (Hrsg.): Global Networks, Linked Cities. - London: Routledge: 309-325.

Clichevsky, N. (2000): Informalidad y segregación urbana en América Latina. Una aproximación. - In: Serie Medio Ambiente y Desarrollo 28, Comisión Económica para América Latina y el Caribe (CEPAL). Dangschat, J. (1997): Sag' mir, wo Du wohnst, und ich sag' Dir, wer Du bist! Zum aktuellen Stand der deutschen Segregationsforschung. - In: Prokla 109, 4: 619-647.

Gilbert,A. (1994): The Latin American City.-London: Latin America Bureau.

Harvey, D. (1989): From managerialism to entrepreneurialism: the transformation in urban governance in late capitalism. - In: Geografiska Annaler 71b, 1: 3-17.

Lungo, M. \& S. Baires (2001): Socio-spatial segregation and urban land regulation in latinamerican cities. - Paper presented at the Seminar «Segregation in the City», Lincoln Institute of Land Policy (LILP), Cambridge, Massachusetts, 25/28 July 2001.

ParnReIter, C. (2001):Transformación socioeconómica y nuevas formas de urbanización en América Latina. In: Burchardt, H. \& H. Dilla (Hrsg.): Mercados globales y gobernabilidad local: retos para la descentralización. - Caracas: Nueva Sociedad: 29-43.

Parnreiter, C. (2002): Mexico: The Making of a Global City? - In: SASSEN, S. (Hrsg.): Global Networks, Linked Cities. - London: Routledge: 145-182.

Portes, A. (1989): Latin American Urbanization During the Years of the Crisis. - In: Latin American Research Review 24: 7-44.

Portes, A., Dore-Cabral, C. \& P. Landolt (Hrsg.) (1997): The Urban Caribbean: Transition to the New Global Economy. - Baltimore: The John Hopkins University Press.

Rubalcava, R. \& M. Schteingart (2000): Segregación socio-espacial en el Área Metropolitana de la Ciudad de México. - In: Garza, G. (Hrsg.): La Ciudad de México en el Fin del Segundo Milenio. - México D.F.: El Colegio de México, Centro de Estudios Demográficos y de Desarrollo urbano, Gobierno del Destrito Federal: 287-296.

Sabatini, F. (2000): Reforma de los mercados de suelo en Santiago, Chile: efectos sobre los precios de la tierra y la segregación residencial. - In: EURE, Revista 
Latinoamericana de Estudios Urbano Regionales 26/77, Santiago de Chile: 49-80.

SABATINI,F., CÁCERES, G. \& J. Cerda (2001): Segregación residencial en las principales ciudades chilenas: Tendencias de las tres últimas décadas y posibles cursos de acción. - In: EURE, Revista Latinoamericana de Estudios Urbano Regionales 27/82, Santiago de Chile: 21-42.

Schteingart, M. (2001): La división social del espacio en las ciudades. - In: Perfiles Latinoamericanos 10,19: 13-31.

TORRES, H. (2001): Cambios socioterritoriales en Buenos Aires durante la década de 1990. - In: EURE, Revista Latinoamericana de Estudios Urbano Regionales 27/80, Santiago de Chile: 33-56.

Wilhelmy, H. \& A. Borsdorf (1984): Die Städte Südamerikas. Teil 1: Wesen und Wandel. - Berlin: Gebrüder Borntraeger.

\section{Zusammenfassung: Transformation und neue Formen der Segregation in den Städten Lateinamerikas \\ Im ersten Teil dieses Artikels wird auf der Basis einer Aufarbeitung der Literatur zum Thema argumentiert, dass sich in den Städten Lateinamerikas in den letzten zwei Jahrzehnten neue Formen sozialräumlicher Segregation herausgebildet haben. Die lange angenom- mene klare räumliche Differenzierung der Wohnge- biete unterschiedlicher sozialer Gruppen entspricht dem komplexen Bild der gegenwärtigen Stadtent- wicklung nicht (mehr). Neue Entwicklungstendenzen deuten auf das Entstehen einer «multi-fragmentier- ten» Stadt hin. Als wesentlicher Grund für das dichtere Nebeneinander von "arm» und «reich» bei gleichzeitig intensiverer Abschottung (u.a. durch gated communities oder condominios) werden die Transformations- und Globalisierungsprozesse ausgemacht, die eine klein- räumigere Segregation über Verarmungs-, Privati- sierungs- und Deregulierungsprozesse fördern.}

Im zweiten Teil des Artikels wird eine Agenda für die condominio-Forschung vorgestellt. Zentrale Punkte bilden einerseits die Frage, warum es zum condominioBoom kommt und wer seine zentralen Akteure sind, und andererseits die Forderung, die condominioForschung tiefer einzubetten in die allgemeine Segregations- und Stadtforschung. Beide Aspekte verweisen auf die Notwendigkeit, den Zusammenhang zwischen Transformationsprozessen und dem Entstehen neuer Formen sozialräumlicher Segregation zu vertiefen.

\section{Summary: Transformation and New Forms of Segregation in the Cities of Latin America}

Based on relevant literature it is argued in the first part of the article that during the past two decades new patterns of socio-spatial segregation in most Latin American cities have emerged. The long-standing assump- tion of clear and large-scale residential segregation patterns does no longer correspond to the complex structure of current urban developments. New studies suggest the emergence of a "multi-fragmented» city. As a result of processes of transformation and globali- . sation (expressed in the liberalization of real estate and urban land markets, impoverishment of households and economic reform in general) the geographical scale of segregation patterns is shifting to a smaller scale - «rich» and "poor» live physically closer to each other. At the same time, the intensity of segregation has increased partly due to the emergence of gated communities or condominios built for social groups outside of their traditional residential areas. For this reason, these communities clearly wall themselves off from their neighbourhood.

In the second part of the article, an agenda for future research on condominios is presented (e.g. factors causing the condominio boom and key actors involved), which has to be better incorporated in segregation and urban studies. Both aspects point to the necessity of investigating the relation between transformation processes and new patterns of residential segregation.

\section{Résumé: Transformation et nouvelles formes de ségrégation dans les villes d'Amérique latine} La première partie du présent article se fonde sur une analyse bibliographique approfondie pour montrer qu'au cours des deux dernières décennies de nouvelles formes de ségrégation socio-spatiale se sont développées dans les villes latino-américaines. La différenciation traditionnelle des espaces résidentiels en fonction de la stratification sociale ne correspond plus à l'image complexe actuelle du développement urbain. De nouvelles tendances de développement mettent l'accent sur l'apparition d'une ville «multi-fragmentée». Les processus de transformation et de mondialisation, qui favorisent des processus de ségrégation, sur des espaces réduits, en matière d'appauvrissement, de privatisation et de dérégulation, sont la cause essentielle d'un voisinage plus dense du «riche» et du "pauvre», parallèlement à l'intensification de la fracture sociale provoquée par le déploiement des gated communities ou condominios.

La seconde partie de l'article est consacrée à l'Agenda de la recherche sur les condominios. Elle pose principalement la question de l'origine du boom des condominios et de ses acteurs centraux, d'une part, d'autre part celle de la nécessité d'intégrer de façon plus systématique la recherche sur les condominios dans la recherche générale sur la ségrégation et la ville. Ces deux aspects mettent l'accent sur la nécessité d'approfondir les rapports entre les processus de transformation et l'apparition de nouvelles formes de ségrégation socio-spatiales. 


\section{Didaktische Hinweise}

- Wie erklärt sich der Begriff Segregation? Welche Arten von Segregation und welche Erscheinungsformen derselben finden sich in Lateinamerika?

- Welches sind die Ursachen und die räumlichen Konsequenzen der ökonomischen und sozialpolitischen Transformation in Lateinamerika?

- Was ist über die condominios in Lateinamerika aus der Forschung bekannt? Welche Fragen bedürfen besonderer Klärung?

Mag. Karin Fischer, Institut für Stadt- und Regionalforschung, Österreichische Akademie der Wissenschaften, Postgasse 7/4/2, A-1010 Wien.

e-mail: Karin.Fischer@oeaw.ac.at

Mag. Dr. Christof Parnreiter, Institut für Stadt- und Regionalforschung, Österreichische Akademie der Wissenschaften, Postgasse 7/4/2, A-1010 Wien.

e-mail: Christof Parnreiter@oeaw.ac.at

\section{Manuskripteingang/received/manuscrit entré le} 11.9.2002

Annahme zum Druck/accepted for publication/accepté pour l'impression: 4.12 .2002 